\title{
A Feasibility Study of an Intervention for Structured Preparation Before Detoxification in Alcohol Dependence: the SPADe Trial Results
}

Christos KOUIMTSIDIS ( $\nabla$ drckouimtsidis@hotmail.com )

Surrey and Borders Partnership NHS Foundation Trust https://orcid.org/0000-0001-9975-2955

\section{Ben Houghton}

Surrey and Borders Partnership NHS Foundation Trust

\section{Heather Gage}

University of Surrey

Caitlin Notley

University of East Anglia School of Health Sciences

Vivienne Maskrey

University of East Anglia School of Health Sciences

Allan Clark

University of East Anglia School of Health Sciences

\section{Richard Holland}

University of Leicester Medical School

Anne Anne Lingford-Hughes

Imperial College London

Bhaskar Bhaskar Punukollu

Camden and Islington NHS Foundation Trust

Morro Morro Touray

University of Surrey

Theodora Duka

University of Sussex

\section{Research}

Keywords: Alcohol dependence, detoxification, structured preparation, pre-habilitation, SPADe

Posted Date: June 2nd, 2020

DOI: https://doi.org/10.21203/rs.3.rs-32361/v1 
License: (c) (i) This work is licensed under a Creative Commons Attribution 4.0 International License. Read Full License

Version of Record: A version of this preprint was published at Pilot and Feasibility Studies on July 29th, 2021. See the published version at https://doi.org/10.1186/s40814-021-00880-6. 


\section{Abstract}

\section{Background}

Individuals who are 'moderately' or 'severely' dependent consume alcohol at levels that are likely to have a severe impact on their own health and mortality, the health and behaviours of others (family members), and to have economic and social implications. Treatment guidelines suggest that treatment needs to be planned with medically assisted withdrawal (also referred to as detoxification), and aftercare support. Treatment outcomes are poor with low proportions engaging in after care and high relapse rates. An approach of structured preparation before alcohol detoxification (SPADe) put emphasis on introducing lifestyle changes, development of coping strategies for cravings, stress and emotions as well as introducing changes to the immediate family and social environment in advance of alcohol cessation. Such a pre-habilitation paradigm compliments the established treatment approach. The key research question was: can we design a large scale, randomised controlled trial (RCT) that will answer whether such an approach is more effective than usual care in helping individuals to maintain longer periods of alcohol abstinence?

\section{Methods}

This is a pragmatic, parallel, two-arm, feasibility RCT comparing the clinical and cost-effectiveness of SPADe and usual care against usual care only in maintaining alcohol abstinence in adults with alcohol dependence receiving care in two community services in London. The trial follows the guidelines of phase 2 of the Medical Research Council (MRC) for complex interventions.

\section{Results}

We were able to recruit 48/50 participants during a period of 9 months. Retention in the trial was $75 \%$. Treatment compliance was overall $44 \%$. Data completion for the primary outcome was $65 \%, 50 \%$ and $63 \%$ at 3,6 and 12 months respectively. The intervention group had more days abstinent in the previous 90 days at the 12 months $(n=54.5)$ versus control $(n=41.5)$.

\section{Discussion}

The results of this feasibility trial indicate that with the appropriate modifications, a full multi centred trial would be possible to test the effectiveness and cost-effectiveness of a pre- habilitation approach such as the SPADe group intervention in addition to usual care against usual care only.

\section{Trial Registration}

Name of registry: ISRCTN; Trial Registration Number: 14621127; Date of Registration: 22/02/2017; URL of trial registry record: http://www.isrctn.com/ISRCTN14621127

\section{Background}


Alcohol harmful use continues to be a global health problem. Tackling the impact of harmful and dependent drinking is a key global public health priority. Global estimates suggest that one in five adults report at least one occasion of heavy episodic drinking in the past month [1]. In the UK hospital admissions attributable to alcohol in 2017-2018 remained similar to the previous year $(338,000)$. However this is $15 \%$ higher than 10 years ago [2]. Alcohol misuse is linked directly to a range of health disorders. Cancer, accidental injuries and mental health problems remain the main alcohol related diagnosis leading to a hospital admission [2]. More than five thousand people died directly from alcohol use in 2016 in the UK, which is 6\% higher than 10 years ago [2]. However in the year 2018-2019, in the UK those in treatment for alcohol alone remained stable as the previous year $(75,787)$. This follows large year-on-year declines from a peak of 91,651 in 2013 to 2014 [3]. Individuals who are 'moderately' or 'severely' dependent consume alcohol at levels that are likely to have a severe impact on their own health and mortality, the health and behaviours of others (family members), and to have economic and social implications [4].

Treatment guidelines in the UK for moderate to severe alcohol dependence have largely remained the same for the past decade. They suggest that treatment needs to be planned, with up to four motivational sessions focusing on treatment engagement and development of aftercare support, followed by medically assisted withdrawal (also referred to as detoxification), and finally aftercare support, which should include pharmacological, psychological and access to self-help interventions and support [4]. Benzodiazepines are normally prescribed during detoxification to reduce the overt symptoms of alcohol withdrawal (sweats, tremor) as well as prevent potentially life-threatening complications (e.g. convulsions, delirium tremens) [4]. However, these drugs do not prevent alcohol craving, relapse back into alcohol drinking and other long-term effects on mental functioning [4,5]. Outcomes from detoxification are often poor with low proportions engaging in after care [6] and high relapse rates [4]. There is accumulating evidence from animal and human studies that exposure to multiple detoxifications is associated, with cognitive and behaviour changes indicating inability in conflict resolution and increased sensitivity to stress, both of which may contribute to relapse and might compromise the effectiveness of aftercare support [7-11]. There is also evidence to suggest that multiple detoxifications can exacerbate craving, adversely impacting on subsequent attempts at achieving abstinence [12].

In the light of this evidence indicating risks associated with the process of detoxification itself, one potential approach to maximise effectiveness of each treatment episode, may be following a prehabilitation treatment paradigm that compliments the established treatment and rehabilitation paradigm. Such an enhanced paradigm takes into account and plans in advance for the management of the risks associated with the detoxification process as well as the risk factors associated with relapse. Such an approach of structured preparation puts emphasis on introducing lifestyle changes, development of coping strategies for cravings, stress and emotions as well as introducing changes to the immediate family and social environment in advance of alcohol cessation. There is currently no guidance specifically on preparation for the detoxification process, apart from general guidance on care coordination and case management [4]. Absence of specific guidance reflects the lack of developed interventions in this area. Evidence is required on whether structured preparation along the lines of pre- 
habilitation principles before detoxification rather than detoxification alone improves treatment outcomes short and long term and therefore whether the whole treatment paradigm should shift. Before embarking on a full trial of the effectiveness of such structured preparation, there is a need to undertake a feasibility study to establish key parameters that influence trial design such as recruitment, adherence to the intervention, retention, and sensitivity of alternative outcome measures.

A literature search of PubMed Central using alcohol relapse prevention treatment related MeSH terms undertaken in June 2014, found (i) that group interventions with diverse theoretical bases are considered to be more cost-effective than one-to-one interventions; and (ii) Cognitive Behaviour Therapy (CBT) Relapse Prevention interventions are well supported by evidence [13].

In the UK an innovative group intervention for preparation before alcohol detoxification, based on CBT Relapse Prevention interventions, developed by members of the research team, reduced detox drop outs [14], and improved outcomes at 1, 3 months (5) and 6 months [15]. However, these findings were from small naturalistic studies. Qualitative evidence found that 'regaining control' was the main learning point across all group sessions of the programme [16].

This feasibility study built on the above preliminary evidence and aimed to refine the preparatory intervention and assess the feasibility of conducting a large scale evaluation (Structured Preparation before Alcohol Detoxification: SPADe) for people with moderate to severe alcohol dependence, as an adjunct to usual care, consisting of planned detoxification and aftercare.

The intervention under investigation in this study is based on Plans, Responses, Impulses, Motives, Evaluations (PRIME) theory of motivation [17] and Learning theories [18] that underlie the use of psychological interventions aiming to reverse the development of automatised behaviour and associated loss of control, such as CBT Relapse Prevention interventions. It combines the long established (in alcohol treatment) ethos of group intervention, and follows the biological principle of homeostasis, which is disturbed with prolonged alcohol use [19], in order to help individuals to regain control over drinking as the first step towards lifelong sustainable abstinence.

\section{Objectives}

The key research question was: can we design a large scale, randomised controlled trial (RCT) that will answer whether the SPADe intervention is more effective than usual care in helping adults to maintain longer periods of alcohol abstinence? The feasibility trial compared the use of SPADe with usual care against usual care alone in the participating sites and enabled us to do the following:

1. 1. Measure the number of eligible participants, willingness of clinicians to recruit participants, recruitment rate, loss to follow-up, adherence to the intervention, and standard deviation of the primary outcome measures. This will ultimately inform the sample size calculation for a multicentre clinical trial. 
2. 2. Determine the acceptability of randomisation to service users, through its effect on recruitment, dropout rates and via qualitative interviews.

3. 3. Determine, through response rates to questionnaires, the appropriateness and the acceptability of the outcome measures to participants, in order to explore the suitability of our chosen secondary outcome measures, that is, percentage of days abstinent, service use and health-related quality of life.

4. 4. Estimate the time needed to collect and analyse baseline and outcome data

5. 5. Explore the utility of the health-related quality of life instrument (EQ-5D-5L) (see outcome measures below) in allowing the estimation of quality adjusted life years in the sample.

Furthermore reduction in subjective measures of alcohol dependence and craving as well as improvement in objective measures of mental functioning were explored. Finally, we have conducted qualitative interviews with participants and service providers, to assess the acceptability of the treatment and to explore their experience of the treatment including any barriers and/ or facilitators to taking part in the study. Findings from these interviews will enable us to refine the SPADe intervention and the design of the future definitive RCT. Methodology and results of the qualitative interviews will be reported in detail separately.

\section{Methods}

\section{Trial Design}

The study design is reported in detail elsewhere [20]. This is a parallel, two-arm, feasibility RCT comparing the clinical and cost-effectiveness of SPADe and usual care, against usual care only, in maintaining alcohol abstinence in adults with alcohol dependence receiving care in the community. The trial follows the guidelines of phase 2 of the Medical Research Council (MRC) for complex interventions [21] and includes: adaptation of the intervention, feasibility study including health economics, and process evaluation. No changes of the protocol trial design has taken place.

\section{Assessments}

Participant assessments were conducted at baseline and at 3, 6 and 12 months by the research assistants (RAs) who were trained in assessing capacity and obtaining consent, promoting the study, administering the questionnaires, and interviewing participants. A window of two weeks at either side of the assessment due points was endorsed in order to maximise engagement with follow up.

\section{Participants}

Inclusion criteria 
1. 1. Presentation to either of the two alcohol services in London (Hounslow, site 1 and Camden, site 2) seeking abstinence from alcohol.

2. 2. Alcohol dependence (moderate to severe), scoring 16 and above on Severity of Alcohol Dependence Questionnaire (SADQ) (see outcome measures below). This level of dependence indicates that it would be clinically appropriate to receive a medically assisted detoxification (4).

3. 3. Stated intention to stay in the area within the time period of the intervention.

4. 4. Willingness to be part of a group intervention if randomised to receive it.

\section{Exclusion criteria}

1. 1. Age less than 18 (as not usually treated by specialist alcohol services).

2. 2. Pregnancy: pregnant women need urgent intervention to withdraw from alcohol, due to the effect of alcohol on the foetus.

3. 3. Known terminal illness with life expectancy of less than 6 months.

4. 4. Severe medical condition that requires urgent medical admission, which would lead to an unplanned medically assisted withdrawal.

5. 5. Severe cognitive impairment that compromises capacity and /or ability to participate in a group intervention.

6. 6. Acute stage of severe and enduring mental illness (schizophrenia, Bipolar Affective Disorder, recurrent depressive disorder: current episode severe), when acute symptomatology compromises service user's ability to participate in a group intervention.

\section{Study Settings}

Participants were recruited from specialist alcohol community services offering recovery orientated treatment for people with Alcohol Use Disorders (AUD). Recruitment took place in two sites in London, both offering the intervention and usual care, in order to explore challenges associated with implementation of the intervention across two different set up of services. Both sites were run by a partnership between a third sector organisation and the National Health Service (NHS), a common funding model in the UK. The two sites had different integration levels; site 1 was fully integrated with a single management and clinical governance system; site 2 had two parallel systems. Furthermore site 1 had been offering the intervention for several years as part of the standard treatment pathway, whereas in site 2 the intervention had to be added to the clinical pathway.

\section{Interventions}

\section{SPADe}

The intervention provides structured group preparation (additional to usual care) with the aim of helping participants (i) regain partial control over their drinking prior to detoxification and (ii) initiation of lifestyle 
changes for the individual and the immediate family environment. These changes are linked with developing new coping skills and enhanced self-efficacy [22].

The six sessions are numbered and offered weekly in a given order. Stabilisation of the amount and pattern of drinking is a common theme across all the sessions. To that effect each session can act as an entry point (i.e. an open rolling programme group), despite the special theme covered in depth during the second part of the session. Each session has two facilitators, lasts for one hour and is divided into three parts:

- - In the first part (15 minutes) group rules are established, new members are introduced, aims of the intervention. In-between sessions practice allocated in previous session where individual targets were set are reviewed.

- - The second part (30 minutes, main part) explores the following themes depending on session number: 1 - Understanding habit, addiction and alcohol dependence; 2 - Stabilise and control your drinking; 3 - Lifestyle changes for you and the people around you; 4 - Reduction of your drinking; 5 - Achieving abstinence; 6 - Relapse prevention strategies.

- - In the third part (15 minutes), the group summarises the main learning points and agrees inbetween sessions practice and targets to be achieved before the next session. A group work folder provided enabling notes and worksheets to be kept together.

The number of participants per group at any point is between two and eight. This is considered appropriate for theory-based treatment groups, to (i) reach a balance between the educational and treatment elements of the intervention, (ii) promote interactions between group members (group therapeutic effect) and (iii) secure the facilitator's attention to each member individually [23]. The duration of 1 hour as suggested in Patient and Public Involvement meetings, reduces the risk of withdrawal symptoms and help participants to maintain concentration.

\section{Usual Care}

Usual Care includes: planning for detoxification; detoxification delivery; and aftercare. Participants entered detoxification at the first available opportunity (expected to be within 4 weeks from presentation). Whilst waiting for detoxification, they meet their keyworker (one-to-one) on 3-4 occasions to maintain motivation and plan aftercare. Participants allocated to the intervention arm entered detoxification at the first available opportunity following completion of the intervention.

Detoxification was medically assisted in the community as an outpatient (both sites), or inpatient (only for site 1), as clinically indicated. The choice depends on health risk factors and availability of social support during detoxification [4]. Furthermore participants allocated to Intervention could achieve abstinence by Guided Self-Detox. Guided Self-Detox refers to gradual reduction of drinking guided by the group facilitator during the intervention period. As mentioned above aftercare (following detoxification) included a relapse prevention group, a small number of individual key worker sessions, pharmacological 
interventions as appropriate peer support groups such as Self-Management and Recovery Training (SMART) Recovery or Alcoholics Anonymous (AA) or more comprehensive aftercare group programme either as an outpatient (both sites) or inpatient (only site 1).

Participants in both intervention and control groups received all the above elements of usual care available in the recruiting service. At any time during the trial, change of clinical needs or risks were monitored by the clinical team and participant's treatment plan was modified accordingly, e.g. urgent hospital admission. These incidents were monitored and reported as per standard ethical recommendations for safety monitoring. Each participant's care pathway was recorded and analysed for variability within and between sites as part of the economic evaluation (see below), to ensure these are equivalent across trial arms.

\section{Outcomes}

Feasibility measures

Feasibility outcomes were: (I) Recruitment and retention rates: monthly monitoring of the number of alcohol dependent clients accessing services during the recruitment period of the study; how many meet the eligibility criteria; and how many were invited and accepted into the study and retained in each group for the full 12 months. (II) Compliance with treatment: number of SPADe sessions attended (for the intervention arm) using the facilitator's record of attendance. (III) Data collection and completeness: attendance for assessments and completeness of instruments; loss to follow up and missing data for all outcomes were analysed.

A variety of possible outcomes were used at different time points (3, 6 and 12 months following randomisation), so the primary outcome for the main trial could be identified and sample size calculations to be conducted, including: duration of continuous abstinence with no incidents of lapse or relapse; percentage of days of abstinence (PDA) (both self-report using Time Line Follow Back method) (TLFB) [24]; time to relapse (from stopping alcohol to first day of alcohol use; also as defined by selfreport).

Secondary outcomes

Secondary outcomes were measured using validated instruments wherever possible at 3, 6 and 12 months:

1. 1. Severity of Alcohol Dependence Questionnaire (SADQ), a 20-item self-completion questionnaire, scores range 0 to 60, (16 to 29 indicates moderate severity, above 30 severe dependence) [25].

2. 2. Alcohol Urge Questionnaire (AUQ), an eight-item self-completion questionnaire containing three domains of drinking urges: desire for a drink; expectation of positive effect from drinking; inability to avoid drinking [26]. 
3. 3. Incentive Conflict Task (ICT). This task assesses inherent conflict in abstaining drinkers, between the intention to abstain from drinking and the desire to drink, that may contribute to relapse [9]. Alcohol-dependent individuals, as they experience successive detoxifications, become increasingly impaired in performing the ICT [27].

4. 4. Euro-QoL (EQ-5D-5L); English version [28], which is a short questionnaire assessing general healthcare used in economic evaluations for the calculation of Quality Adjusted Life Years.

5. 5. Self-reported participation in aftercare activities, using a specifically developed log, measuring type and frequency of activity attended, during the period prior the follow up interview.

Process evaluation - treatment fidelity

Observation of twenty five percent of the intervention sessions, offered across both recruitment sites, during the whole duration of the studywas planned using the Yale Adherence and Competence Scale (YACS II) (2005) [29]. Furthermore, $10 \%$ of the sessions was planned to be rated by an additional independent rater using YACS II. Group facilitators were asked to complete a self-assessment form following each session to reflect on their fidelity to the intervention manual.

Audio recording and rating of twenty five percent of the key-working sessions offered to the usual care group was plannedin order to assess possible contamination between the study arms, using a specifically developed form based on YACS II items and the main objectives of the SPADe group intervention with low score indicating no presence of specific CBT content and no contamination.

\section{Sample Size}

No formal calculation is required for a feasibility study since measuring effectiveness is not a key objective. The sample size of 50 was considered appropriate for assessing key objectives related to recruitment, retention, randomisation, data capture, performance of outcome measures and acceptability.

\section{Randomization And Masking}

Once consented, participants were randomised using a third-party web-based randomisation system which ensured concealed allocation. Participants were stratified according to number of previous detoxifications (> 2 vs $\leq 2$ ) and site. Randomisation had a random block size (2-4). Research Assistants (RAs) were blind to the randomisation of the opposite study site. It was planned to for them to conduct the follow-up interviews of the opposite site. This initial plan for cross site follow up was abandoned early during the 3 months follow up period as it was proven administratively challenging and was considered a barrier for successful follow up efforts.

\section{Statistical And Health Economic Analysis}


The main analysis was planned to be based on the intention-to-treat principle considering all randomised clients according to the arm they were allocated.

The feasibility outcomes were summarised using descriptive statistics where appropriate. The potential primary and secondary outcomes were summarised by arm, as well as completion rates estimated for each outcome measure. Duration of continuous abstinence, as measured from randomisation, and the time to relapse, as measured from the end of the detoxification wereanalysed using a Kaplan-Meier curve and log-rank test. The percentage of Days of Abstinence (PDA) were analysed using a regression model. Formal hypothesis tests and confidence intervals were conducted although due to the nature of this trial they were treated cautiously and the main focus was on the completion rate of outcomes, and the estimation of parameters required for a sample size calculation for the main trial.

Economic evaluation in the feasibility study tested the collection of data on costs and outcomes that would be required in any future definitive trial assessing the cost effectiveness of the SPADe intervention. Clinic contacts with key workers and doctors for delivering the intervention and usual care were obtained retrospectively from clinic records by research assistants. To assess potential service use offsets, data on other service utilisation by participants was gathered by self report using the Client Service Receipt Inventory. The CSRI was administered to participants by researchers in the clinic at 3, 6 and 12 months. The questionnaire covers all forms of health and social care, and includes contacts with the police and justice services [30].

\section{Qualitative Interviews}

A purposive sample of participants across both trial groups and sites (approximately 20) were planned to be interviewed at three months to establish experiences of randomisation, recruitment and initial trial procedures (wave 1) and follow up interviews were planned to take place at 9 months (completion of the study, wave 2) to give specific feedback on retention issues and treatment conditions. Due to difficulties interviewing participants on two time points, interviews were conducted at any time between 6 and 9 months since recruitment into the study. A selected sample of staff involved with the intervention (both directly and in-directly) were interviewed on study completion.

\section{Results}

\section{Demographics}

We recruited 48 out of 50 alcohol dependent individuals, aged 18 or over who had a desire to stop drinking. Table 1 shows the baseline data for all 48 randomised participants. Overall, whilst the two groups were reasonably equivalent in terms of mean age, ethnicity, years of education and key variables such as baseline SADQ, there were also important differences in sex and living arrangements with more males and single people in the intervention group. However, this is not unexpected given the size of the groups. Overall, the participants had an average age of 46.4 years (range: 31 to 63 ), 28 (58\%) were male 
and the had previously undertaken and median of 2 previous detoxifications (range: 0 to 24). These demographics are similar to the population in treatment 
Table 1

Baseline characteristics of randomised participants.

\begin{tabular}{|c|c|c|}
\hline & Usual Care $(n=23)$ & Intervention $(n=25)$ \\
\hline Age (years), mean (SD) & $46.70(9.82)$ & $46.16(10.94)$ \\
\hline \multicolumn{3}{|l|}{ Gender, $\mathrm{n}(\%)$} \\
\hline Female & 13 (57\%) & $7(28 \%)$ \\
\hline Male & $10(43 \%)$ & $18(72 \%)$ \\
\hline \multicolumn{3}{|l|}{ Ethnicity, n(\%) } \\
\hline African & $1(4 \%)$ & $0(0 \%)$ \\
\hline Any other Black background & $1(4 \%)$ & $1(4 \%)$ \\
\hline Any other ethnic group & $0(0 \%)$ & $1(4 \%)$ \\
\hline Any other white background & $3(13 \%)$ & $1(4 \%)$ \\
\hline British & $12(52 \%)$ & $14(56 \%)$ \\
\hline Carribbean & $0(0 \%)$ & $1(4 \%)$ \\
\hline Indian & $5(22 \%)$ & $3(12 \%)$ \\
\hline Irish & $1(4 \%)$ & $3(12 \%)$ \\
\hline Pakistani & $0(0 \%)$ & $1(4 \%)$ \\
\hline Migrant, n(\%) & $11(48 \%)$ & $9(36 \%)$ \\
\hline Years of education, mean (SD) & $11.57(2.09)$ & $11.88(2.39)$ \\
\hline \multicolumn{3}{|l|}{ Highest qualification, n(\%) } \\
\hline A Level / NVQ & $2(9 \%)$ & $7(28 \%)$ \\
\hline Diploma / BTEC & $2(9 \%)$ & $0(0 \%)$ \\
\hline No qualification & $4(17 \%)$ & $3(12 \%)$ \\
\hline O level / GCSE & $11(48 \%)$ & $9(36 \%)$ \\
\hline Other (please specify) & $1(4 \%)$ & $0(0 \%)$ \\
\hline University Degree & $3(13 \%)$ & $6(24 \%)$ \\
\hline \multicolumn{3}{|l|}{ Employment status, n(\%) } \\
\hline Full time & $4(17 \%)$ & $3(12 \%)$ \\
\hline Part time & $2(9 \%)$ & $3(12 \%)$ \\
\hline
\end{tabular}




\begin{tabular}{|c|c|c|}
\hline & Usual Care $(n=23)$ & Intervention $(n=25)$ \\
\hline Sick leave $>4$ weeks & $4(17 \%)$ & $2(8 \%)$ \\
\hline Unemployed & $13(57 \%)$ & $17(68 \%)$ \\
\hline \multicolumn{3}{|l|}{ Relationship status, n(\%) } \\
\hline Divorced & $2(9 \%)$ & $3(12 \%)$ \\
\hline In a relationship but not living together & $3(13 \%)$ & $4(16 \%)$ \\
\hline Living together & $1(4 \%)$ & $1(4 \%)$ \\
\hline Married & $6(26 \%)$ & $1(4 \%)$ \\
\hline Separated & $1(4 \%)$ & $1(4 \%)$ \\
\hline Single & $9(39 \%)$ & $15(60 \%)$ \\
\hline Widowed & $1(4 \%)$ & $0(0 \%)$ \\
\hline Family history of alcohol problems, $n(\%)$ & $12(52 \%)$ & $12(48 \%)$ \\
\hline \multicolumn{3}{|l|}{ Previous detoxification ${ }^{1}$, median (IQR) } \\
\hline Total number & $2(1,3)$ & $1(1,5)$ \\
\hline Inpatient alcohol unit & $0(0,0)$ & $0(0,1)$ \\
\hline Acute hospital & $0(0,0)$ & $0(0,0)$ \\
\hline Community or outpatient & $0(0,1)$ & $0(0,0)$ \\
\hline Stopping without medication & $1(0,2)$ & $1(0,3)$ \\
\hline Current smoker, n(\%) & $14(61 \%)$ & $20(80 \%)$ \\
\hline Used illicit drugs, n(\%) & $6(26 \%)$ & $8(32 \%)$ \\
\hline Mental health condition, $\mathrm{n}(\%)$ & $14(61 \%)$ & $13(52 \%)$ \\
\hline Current medication ${ }^{2}$ & $11(48 \%)$ & $11(44 \%)$ \\
\hline Psychological therapy in previous 6 months, $n(\%)$ & $3(13 \%)$ & $3(12 \%)$ \\
\hline SADQ, mean (SD) & $34.61(12.93)$ & $33.59(10.45)$ \\
\hline \multicolumn{3}{|l|}{ SADQ Group } \\
\hline $0-29$ & $8(35 \%)$ & $8(36 \%)$ \\
\hline $30+$ & $15(65 \%)$ & $14(64 \%)$ \\
\hline Alcohol Urge Questionnaire, mean (SD) & $35.30(17.04)$ & $37.62(14.19)$ \\
\hline
\end{tabular}




\section{Feasibility outcomes}

\section{Recruitment}

We were able to recruit 48/50 participants during a period of 9 months (September 2017-May 2018). Thirty-three participants were recruited from site 1 and 15 from site 2. The main reasons for declining participation included not wishing to be abstinent and inability to commit to group work. Recruitment was easier when the research assistants were on site, which was supported by the qualitative interviews of staff (see section below).

Retention

Retention in the trial was overall $75 \%, 80 \%(n=20)$ for the intervention group and $69 \%(n=16)$ for usual care. The retention rate indicates strong acceptability in the study population.

\section{Follow up}

Overall the follow up rate was $65 \%(n=31)$ at three months, $50 \%(n=24)$ at six months and $63 \%(n=30)$ at 12 months, with more follow ups taking place over the phone. The follow up rate at 3 months was $74 \%$ $(n=17)$ for usual care and $56 \%(n=14)$ for the intervention. At 6 months it was $52 \%(n=12)$ for usual care and $48 \%(n=12)$ for the intervention. Finally at 12 months it was $61 \%(n=14)$ for usual care and $64 \%(n=16)$ for the intervention. (Fig. 1)

Intervention sessions attended

Treatment compliance, defined as attending 6 sessions over 12 weeks, was overall $44 \%$ with $47 \%(8 / 17)$ and $37.5 \%$ (3/8) for the first and second site respectively, which is considered acceptable for population attending addiction services [13].

\section{Data completeness}

Completeness of data outcomes was varied ( $29 \%$ as lowest for Alcohol Urge Questionnaire at 6 months to $74 \%$ for days of abstinence at 3 months). Completeness of primary outcome (PDA) was $65 \%, 50 \%$ and $63 \%$ at 3,6 and 12 months follow up respectively.

Completion of the Incentive Conflict Task (ICT) was compromised with only 13 tests completed at 3 months and even less at all 3 follow up points. This was direct result of the need for most participants to be follow up over telephone and not face to face as initially planned. 
Table 2

Feasibility outcomes

\begin{tabular}{|c|c|c|c|}
\hline Outcome & $\begin{array}{l}\text { Control }(n= \\
23)\end{array}$ & $\begin{array}{l}\text { Intervention ( } n= \\
25)\end{array}$ & $\begin{array}{l}\text { Overall }(n= \\
48)\end{array}$ \\
\hline $\begin{array}{l}\text { Recruitment rate, number per month }(95 \% \\
\mathrm{Cl})\end{array}$ & & & $6(4.42,7.96)$ \\
\hline Trial retention rate & $16(69.6 \%)$ & $20(80.0 \%)$ & $36(75.0 \%)$ \\
\hline \multicolumn{4}{|l|}{ Compliance } \\
\hline Site 1 & & 8 / 17 (47.0\%) & \\
\hline Site 2 & & 3 / 8 (37.5\%) & \\
\hline \multicolumn{4}{|l|}{ Completeness } \\
\hline \multirow{2}{*}{\multicolumn{4}{|c|}{$\begin{array}{l}\text { Time line } \\
\text { (Days of abstinence) }\end{array}$}} \\
\hline & & & \\
\hline 3 Months & $17(74 \%)$ & $14(56 \%)$ & $31(65 \%)$ \\
\hline 6 Months & $12(52 \%)$ & $12(48 \%)$ & $24(50 \%)$ \\
\hline 12 Months & $14(61 \%)$ & $16(64 \%)$ & $30(63 \%)$ \\
\hline \multicolumn{4}{|l|}{ SADQ } \\
\hline 3 Months & $15(65 \%)$ & $14(56 \%)$ & $29(60 \%)$ \\
\hline 6 Months & $7(30 \%)$ & $8(32 \%)$ & $15(31 \%)$ \\
\hline 12 Months & $12(53 \%)$ & $13(52 \%)$ & $25(52 \%)$ \\
\hline \multicolumn{4}{|l|}{ Alcohol Urge } \\
\hline 3 Months & $15(65 \%)$ & $13(52 \%)$ & $28(58 \%)$ \\
\hline 6 Months & $7(30 \%)$ & $7(28 \%)$ & $14(29 \%)$ \\
\hline 12 Months & $11(48 \%)$ & $11(44 \%)$ & $22(46 \%)$ \\
\hline
\end{tabular}

Progression criteria to a full RCT were agreed in advance and included study retention, treatment compliance and data completion. As shown in Table 3, retention was excellent and no change is required. Regarding treatment compliance minor changes are required, whereas regarding data completion for primary outcome major changes are required before proceeding to a full trial. 
Table 3

Study progression criteria

\begin{tabular}{|llll|}
\hline & Proceed & $\begin{array}{l}\text { Proceed } \\
\text { with } \\
\text { changes }\end{array}$ & $\begin{array}{l}\text { Do not proceed } \\
\text { without major changes }\end{array}$ \\
\hline Compliance with the intervention & $>=60 \%$ & $40-59 \%$ & $<40 \%$ \\
\hline Retention of participants in trial & $>=75 \%$ & $50-74 \%$ & $<50 \%$ \\
\hline $\begin{array}{l}\text { Completion of primary outcome data in } \\
\text { participants not lost-to-followup }\end{array}$ & $>=90 \%$ & $80-89 \%$ & $<80 \%$ \\
\hline
\end{tabular}

\section{Primary Outcomes}

The intervention had more days abstinent in the previous 90 days at the 12 months (54.5) versus usual care (41.5). Seven participants of the intervention and 5 of the usual care group restarted drinking at some point during the follow up period.

\section{Secondary outcomes}

Severity of dependence (SADQ scores) were higher, time to relapse shorter and urges (Alcohol Urge Questionnaire scores) higher for the intervention group at all follow up points.

Equal percentages (52\%) from each arm were detoxed (including guided self-detox). This is lower than anticipated, possibly due to generic challenges that services face.

\section{Health economics evaluation}

Of the total of 48 participants, 39 were included in the health economic analysis ( 19 intervention and 20 usual care). Of the nine excluded, one had crossed groups and the clinic records for eight in one site were missing due to a facility relocation. Clinic data were not complete for many of the remaining 39 participants with more items missing in the usual care than intervention group. Approximately $50 \%$ of participants in both groups provided 12 month follow up questionnaire data (service use and EQ-5D). These levels of missing data were considered too high to provide accurate estimates of costs or changes in health related quality of life. Two key workers delivered each session of the intervention at a cost (2018) of $£ 102$ per session ( $£ 17$ per participant per session, based on six per group, and NHS unit costs [31].

\section{Process evaluation-treatment fidelity}

At least one of each of six group sessions were observed and scored for compliance with the manual, by the Chief Investigator (CI) and an independent rater, using YACS II (2005). Furthermore group facilitators were interviewed by the chief investigator following end of intervention provision period for the study. Tape recording of key working sessions offered as part of usual care was done in site 1 only. Nine 9 sessions assessed in total, with at least one of each of three planned sessions and at least one per keyworker, using a specifically developed tool (see above). In the second site keyworkers refused to be 
taped. To that effect medical notes were reviewed by $\mathrm{Cl}$ and content of session was scored using the same assessment tool. Scores suggested no contamination of key working session with content from the intervention in site 1. In site 2 though, $3 / 7$ participants allocated to usual care, have received additional sessions of psychological work beyond Motivational Interviewing, either by their keyworker or a psychologist. .

Qualitative interviews

Fourteen participants were interviewed from both sites. Five of those were interviewed twice (between 3, 6 and 12 months) as per the original protocol, providing in total 19 interviews. Overall the qualitative data was generally positive and supportive of the study. With a few exceptions, participants understood the purpose of the study and were willing to be randomised. It was noteworthy that there was some confusion regarding group allocation. This suggests that simply 'being in the study' had a positive benefit. Also of note was the positive feedback for the preparation groups (intervention). However the interviews gleaned very little detailed data on CBT specific elements of the group intervention. Instead, participants had generally positive feedback that seemed to be related to a generalised group effect (being part of a group, sharing experiences, and learning from others). Most people found this beneficial, with only a few exceptions (notably those with diagnosed mental health conditions who found group participation difficult).

Two staff members were interviewed (one from each site) following the end of the study. Staff were generally supportive of the intervention, however staff acceptability of both content and structure was key in consistent facilitation of the intervention. Staff were initially hesitant to use novel pre habilitation strategies until they had seen results for themselves and then understood how it was useful to participants. There were some practical issues raised - availability of rooms and room set up as being important to the running of the groups. There was a lot of discussion about retendering and outcome measures for joint service providers. These discussions initially impeded smooth service running and group facilitation until it was evident that the intervention facilitated outcome measures for the service providers. This is an important context for understanding difficulties with implementing the group intervention.

\section{Discussion}

We report here the results of a feasibility study on the addition of structured preparation before alcohol detoxification to usual care against usual care only. The effect of adding some psychological intervention during detoxification have been investigated before [32]. This study investigates the feasibility of enhancing further the psychological work offered before detoxification within the overall concept of prehabilitation, to complement the existing rehabilitation treatment approach.

We were able to recruit appropriate participants (moderate to severe dependence on alcohol) with a rate of 6 recruits per month. The sample demographic characteristics were similar to those of the population in treatment [33]. Randomisation was successful with the exception of more males and single people 
having been allocated to the intervention arm, however, neither were considered to be important confounders likely to results of this feasibility trial. Nevertheless, stratification based on gender and living arrangements will be considered for the future trial.

The retention rate was very good for this type of population. Similarly follow up rates at 12 months for primary outcome were also acceptable. Low follow up rates were observed at 6 months which coincided with the immediate period prior and post re-tendering in the highest recruiting site (site 1). Interview over telephone was the best follow up strategy but compromised the ability to collect data for ICT, CSRI and other questionnaires, indicating the importance of using brief tools rather than self-completion questionnaires. A mixed method of CSRI and clinical notes review should be used for health evaluation. There were also technical difficulties with the operation of ICT. Despite the above challenges this is the first time that ICT is used with persons while they are still drinking (23 participants at baseline), which provides valuable data on the effect of alcohol in decision making (see Table 2). These results will be reported separately. Data completion though for the primary outcome was lower than expected suggesting major changes required. A longer recruitment period with full time RAs positioned in each recruiting site is considered more effective rather than seeking larger numbers of recruiting sites with either part time RAs or full time RAs recruiting for shorter recruitment periods.

To allow for better monitoring of study participants RAs were only blind to randomisation of the opposite site. The original plan was for RAs to complete follow ups of the opposite site hence to be blind to allocation. This was proven over ambitious for a trial with this population. The administrative arrangements and the resulted limited flexibility of RAs due to travel arrangements compromised the initial follow ups. Blinding for psychological interventions is difficult with this population [4].

Compliance with the intervention although acceptable, has indicated that changes are required for the full trial. We consider that recruitment and randomisation at the first point of contact with the service has increased the risk of early dropping out of treatment as a whole, compromising treatment compliance. Furthermore in the current study two clients, with long histories of poor treatment retention and early drop outs were allocated to the intervention arm. In a future RCT, we will randomise clients at a later point, once they are retained for a brief period (usual practice 4 weeks). In a future RCT, the intervention will be better standardised with expectation that participants attend consecutive weekly sessions, within a maximum period of 12 weeks, with missed sessions offered by one of the facilitators as individual brief sessions and more enhanced cooperation between key workers and group facilitators.

The strategy used to assess fidelity to the intervention manual was adequate. It seems that the training, supervision and support provided to group facilitators were adequate for the feasibility trial. For the future trial this task should be allocated to local supervisors per recruitment sites for more hands-on support. This is considered even more important for the keyworkers to reduce even further any contamination between the intervention and usual care.

There was a major difference between the two sites on the procedures for access to inpatient detoxification. In site 1 the decision and the budget was controlled by the local team whereas in site 2 the 
decision and budget allocated involved commissioners and specific steps had to be followed (which did not allow randomisation). This meant that only participants requesting outpatient detoxification were entered into the study in site 2 . This number was anticipated and proven to be low. The rest of the procedures across sites were standardised during phase 1 .

The low rate of participants receiving detoxification for both arms, could be an indicator of limited progression of clients through treatment stages, which might indicate major challenges that services face in their efforts to deliver treatment in a timely and structured way. Generic factors such as tendering of services, limited resources and high turnover of staff, could compromise the capacity of treatment services and systems to host clinical research and could also compromise the standarised delivery of both intervention and usual care. These factors have been discussed widely both in the past and more recently in the UK [32]. In a future trial the ongoing support to group facilitators and the monitoring of usual care provision should be augmented, given the challenging clinical reality.

\section{Conclusions}

Psychological interventions with people who are dependent on alcohol and who are actively drinking at the time of the intervention have been regarded challenging. Furthermore limited work has been done so far on their effectiveness and cost-effectiveness. The results of the feasibility trial indicate that despite the challenges described above, with the appropriate modifications, a full multi centred trial would be possible to test the effectiveness and cost-effectiveness of a pre- habilitation SPADe group intervention against usual care.

\section{Declarations}

\section{Ethics approval and consent to participate}

The study was approved by the Health Research Authority (HRA) Research Ethics Committee (REC) (IRAS ID:213086) for all participating centres. All study personnel will comply with the MCA 2005 (33) and published research governance guidelines. We anticipate that all participants will have capacity to consent and sufficient verbal communication skills to take part in the treatment and in the qualitative interview. Informed written consent will be obtained from all participants. As this is a non-invasive intervention, we do not anticipate any adverse events, but we will follow safety reporting guidance issued by the National Research Ethics Service (UK) for studies except clinical trials of investigational medicinal products. The study has been approved by the Research and Development (R\&D) departments of the relevant recruiting sites.

\section{Consent for publication}

Not applicable 


\section{Availability of data and materials}

The datasets generated and/or analysed during the current study are available from the corresponding author on reasonable request.

\section{Competing interests}

The authors declare that they have no competing interests.

\section{Funding}

This study is supported by the UK National Institute for Health Research (NIHR) under its Research for Patient Benefit (RfPB) Programme (Grant Reference Number PB-PG-0815-20014). The funder of the study had no role in study design, data collection, data analysis, data interpretation, or writing of the report. The authors had full access to all the data in the study and had final responsibility for the decision to submit for publication.

\section{Authors' contributions}

CK had overall management of the research project, developed the intervention manual,supervised the therapists and contributed to all outputs such as papers in conference and journals. He has contributed to the writing of the current paper.

$\mathrm{BH}$ is a Research assistant on the project, conducted the assessments and interviews with participants and carers, has contributed to the paper content and its preparation for submission.

HG is the lead for the health economics part of the project and oversaw the analysis of those data.

$\mathrm{CN}$ is the lead for the qualitative study. She trained the research assistants for the qualitative interviews and supervised the analysis of the data.

VM was the overall project manager. She was responsible for the coordination of study recruitment across sites, the supervision and support of the research assistants.

AC is the project statistician. He was responsible for, all statistical aspects including randomisation and analysis of quantitative data.

$\mathrm{RH}, \mathrm{ALH}$ contributed into the methodological aspects of the project and participated in data analysis and dissemination of the findings.

BP was responsible for coordination of recruitment from one of the participating services. 
TD was responsible for the training and appropriate use of the ICT.

\section{Acknowledgements}

We thank the service users' representatives participating in this study. We thank the study sponsor Surrey and Borders Partnership NHS Foundation Trust, the Camden and Islington NHS Foundation Trust, collaborating researcher assistant and therapists, and members of the Steering Committees.

This paper presents independent research funded by the National Institute for Health Research (NIHR) under its Research for Patient Benefit(RfPB) Programme (Grant Reference Number PB-PG-0815-20014). The views expressed are those of the author(s) and not necessarily those of the NIHR or the Department of Health and Social Care.

\section{Authors' information}

$\mathrm{CK}$ is a Consultant Psychiatrist in Addictions; his $\mathrm{PhD}$ is on psychological interventions in substance misuse. $\mathrm{BH}$ is a research assistant. HG is a Professor of Health Economics. CN is a Senior Lecturer in Mental Health and a Research fellow of the Society for the Study of Addiction. VM is a Research Fellow and Senior Programme Co-ordinator. AC is a Senior Lecturer in Statistics. RH is Head of Leicester Medical School and Professor of Public Health Medicine. ALH is a Professor in Psychiatry, Chair of Addiction Biology and Consultant Psychiatrist in Addictions. BP is a Consultant Psychiatrist in Addictions. TD is a Professor in Experimental Psychology.

\section{References}

1. [1] Peacock A, Leung J, Larney S, Colledge S, Hickman M, Rehm J, Giovino GA, West R, Hall W, Griffiths P, Ali R, Gowing L, Marsden J, Ferrari AJ, Grebely J, Farrell M, Degenhardt L. Global statistics on alcohol, tobacco and illicit drug use: 2017 status report. Addiction 2018; 113: 1905-1926.

2. [2] Public Health England. Statistics on Alcohol 2019. Available on https://digital.nhs.uk/data-andinformation/publications/statistical/statistics-on-alcohol/2019/part-1

3. [3] Adult substance misuse treatment statistics 2018 to 2019: report. November 2019. Available on : https://www.gov.uk/government/publications/substance-misuse-treatment-for-adults-statistics2018-to-2019/adult-substance-misuse-treatment-statistics-2018-to-2019-report

4. [4] Alcohol use disorders: diagnosis, assessment and management of harmful drinking and alcohol dependence. London: NICE, 2010.

5. [5] Lingford-Hughes AR, Welch S, Peters L, Nutt D. BAP updated guidelines:evidence-based guidelines for the pharmacological management of substanceabuse, harmful use, addiction and comorbidity: recommendations from BAP.J Psychopharmacol (2012) 26(7):899-952. doi:

$10.1177 / 0269881112444324$. 
6. [6] Kouimtsidis C, Drable K, Ford L. Implementation and evaluation of a three stages community treatment programme for alcohol dependence. A short report. Drugs: Education, Prevention and Policy 2012; 19 (1), 81-3.

7. [7] Kouimtsidis C, Duka T, Palmer E, Lingford-Hughes A. Prehabilitation in Alcohol Dependence as a Treatment Model for Sustainable Outcomes. A Narrative Review of Literature on the Risks Associated WithDetoxification, From Animal Models to Human Translational Research. Frontiers in Psychiatry; 2019. doi:10.3389/fpsyt.2019.00339

8. [8] Duka T, Townshend JM, Collier K, Stephens DN. Impairment in Cognitive Functions After Multiple Detoxifications in Alcoholic Inpatients. Alcoholism: Clinical and Experimental Research 2003;27(10):1563-72.

9. [9] Loeber S, Duka T, Welzel H, Nakovics H, Heinz A, Flor H, et al. Impairment of Cognitive Abilities and Decision Making after Chronic Use of Alcohol: The Impact of Multiple Detoxifications. Alcohol and Alcoholism 2009; 44(4):372-81.

10. [10] Duka T, Trick L, Nikolaou K, Gray MA, Kempton MJ, Williams H, et al. Unique Brain Areas Associated with Abstinence Control Are Damaged in Multiply Detoxified Alcoholics. Biol Psychiatry. 2011; 70(6): 545-552.

11. [11] Loeber S, Duka T, Welzel Márquez H, Nakovics H, Heinz A, Mann K, et al. Effects of Repeated Withdrawal from Alcohol on Recovery of Cognitive Impairment under Abstinence and Rate of Relapse. Alcohol and Alcoholism. 2010;45(6):541-7.

12. [12] Duka T, Townshend JM, Collier K, Stephens DN. Kindling of Withdrawal: A Study of Craving and Anxiety After Multiple Detoxifications in Alcoholic Inpatients. Alcoholism: Clinical and Experimental Research. 2002;26(6):785- 95

13. [13] Raistrick D, Heather N, Godfrey, C. Review of the effectiveness of treatment for alcohol problems. London: National Treatment Agency for Substance Misuse; 2006.

14. [14] Kouimtsidis C, Ford L. A staged programme approach for alcohol dependence: Cognitive Behaviour Therapy groups for detoxification preparation and aftercare; preliminary findings. Drugs: education, prevention and policy. 2011;18(3):237-9.

15. [15] Kouimtsidis C, Sharma E, Smith A, Charge KJ. Structured intervention to prepare dependent drinkers to achieve abstinence; results from a cohort evaluation for six months post detoxification. Journal of Substance Use, 2015; DOI: 10.3109/14659891.2015.1029020.

16. [16] Croxford A, Notley C, Maskrey V, Holland R, Kouimtsidis C. An exploratory qualitative study seeking participant views evaluating group Cognitive Behavioural Therapy group preparation for alcohol detoxification. Journal of Substance Use. 2015; 20(1), 61-68.

17. [17] West R. Theory of addiction. London: Blackwell Publishing; 2006.

18. [18] Bandura A. Self-Efficacy in changing societies. London: Cambridge University Press; 1995.

19. [19] Koob G.F. \& leMoal M. Drug Abuse: Hedonic Homeostatic Dysregulation. Science. 1997; 278, 5258 
20. [20] Kouimtsidis, C., Houghton, B., Gage, H. et al. A feasibility study of an intervention for structured preparation before detoxification in alcohol dependence: the SPADe trial protocol. Pilot Feasibility Stud 5, 59 2019. https://doi.org/10.1186/s40814-019-0446-1

21. [21] Craig P, Dieppe P, Macintyre S, Michie $S$ et al. Developing and evaluating complex interventions: the new Medical Research Council guidance. British Medical Journal. 2008; 337: a1655.

22. [22] Kouimtsidis C, Charge KJ, Moch J, Stahl D.. Abstinence Preparation Group Intervention for dependent alcohol users. How does it work? Results of a process study. Journal of Substance Use. 2017; 22(2): 149-155. DOI: 10.3109/14659891.2016.1153164.

23. [23]Bieling P, McCabe, R.E, \& Antony, M.M. Cognitive-Behavioral Therapy in Groups. New York: Guilford Press; 2006.

24. [24] Sobell, L.C. \& Sobell, M.B.. Timeline Follow-back: A technique for assessing self-reported ethanol consumption. In J. Allen \& R. Z. Litten (Eds.), Measuring Alcohol Consumption: Psychosocial and Biological Methods 1992; 41-72. Totowa, NJ: Humana Press.

25. [25] Stockwell T, Murphy D, Hodgson R. The Severity of Alcohol Dependence Questionnaire: Its use, reliability and validity. British Journal of Addiction. 1983; 78: 145-155.

26. [26] Bohn M.,Krahn DD, Stachler BA. Development and initial validation of a measure of drinking urges in abstinent alcoholics. Alcoholism: Clinical and Experimental Research. 1995; 19: 600-606.

27. [27] Duka T, Stephens DN. Repeated Detoxification of Alcohol-Dependent Patients Impairs Brain Mechanisms of Behavioural Control Important in Resisting Relapse. Curr Addict Rep. 2014; 1: 1-9. DOI 10.1007/s40429-013-0009-0

28. [28] EQ-5D-5 L User Guide. Basic information on how to use the EQ-5D-5 L instrument. https://euroqol.org/wp-content/uploads/2016/09/EQ-5D-5L_UserGuide_2015. pdf Accessed July 2017

29. [29] Nuro, K.F., Maccarelli, L., Baker, S.M., Martino, S., Rounsaville, B.J., Carroll, K.M. (2005). Yale Adherence and Competence Scale (YACS-II) guidelines. Yale University Psychotherapy Development Center. Substance Abuse Center, West Haven.

30. [30]Chisholm D, Knudsen HC, Amaddeo F, Gaite L, van Wijngaarden B, Epsilon

31. study group. Client socio-demographic and service receipt inventory -

32. European version : development of an instrument for international research.

33. Br J Psychiatry 2000; 177: s28-s33. doi: https://doi.org/10.1192/bjp.177.39.s28.

34. [31] Curtis L. Unit costs of health and social care 2017. www.pssru.ac.uk

35. Alwyn T, John B, Hodgson RJ, Phillips CJ. The addition of a psychological intervention to a home detoxification programme. Alcohol Alcohol. 2004 Nov-Dec;39(6):536-41.

36. [32] https://www.gov.uk/government/statistics/substance-misuse-treatment-for-adults-statistics2018-to-2019

37. [33] Bowden-Jones O, Sinclair J, Lingford-Hughes A. Psychiatry and the global drugs debate: what every psychiatrist needs to know. British Journal of Psychiatry. 2020. DOI: 
Figures

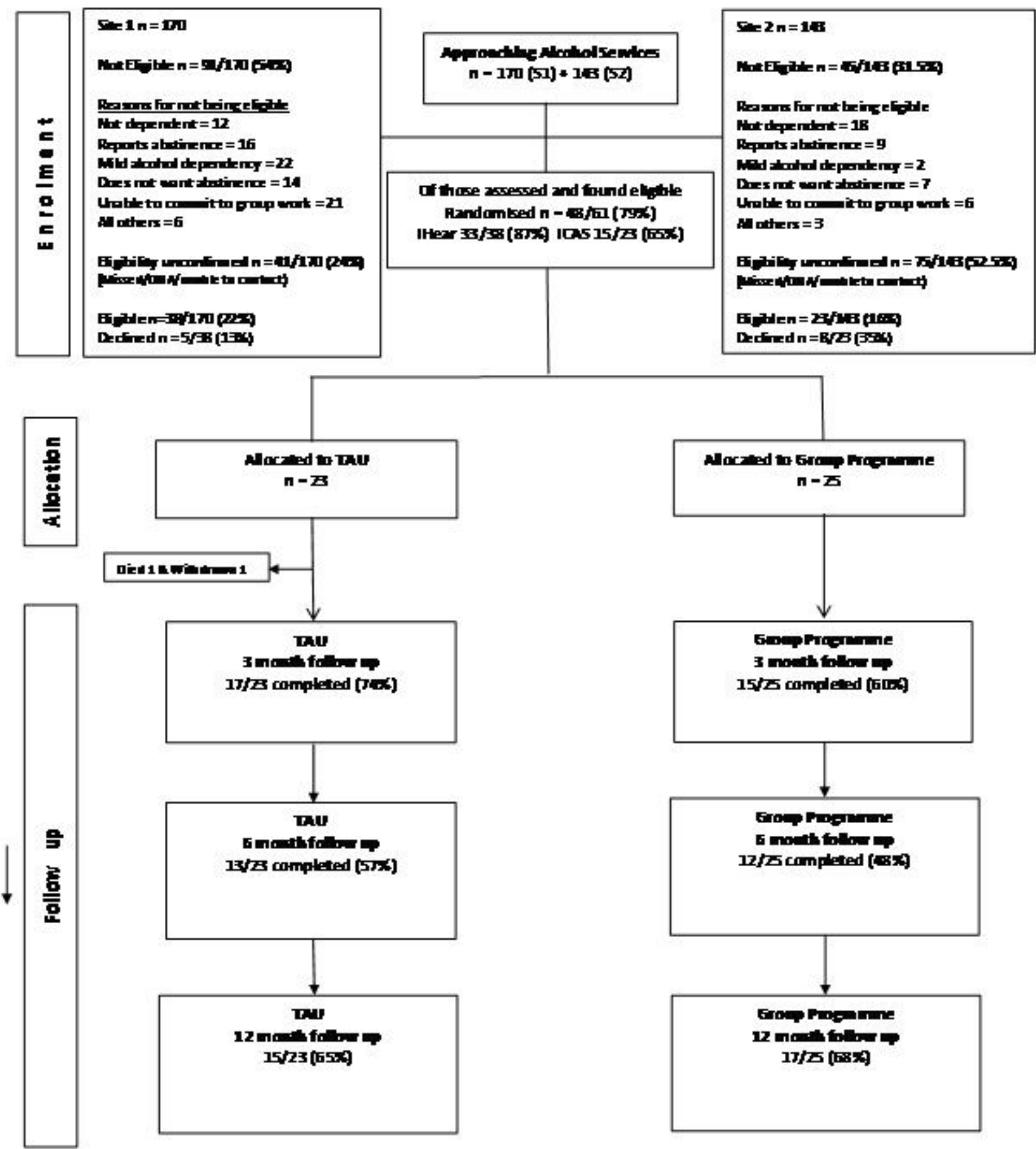

Figure 1

SPADe Feasibility Study Consort Diagram (by Treatment Arm) 